\title{
ON METHODOLOGY OF CONSTRUCTING OF PHYSICAL THEORIES
}

\section{JAROSŁAW KACZMAREK}

Institute of Fluid-Flow Machinery

Polish Academy of Sciences

80-231 Gdańsk, ul. J. Fiszera 14

Poland

e-mail: jarekk@imp.gda.pl

\begin{abstract}
In this paper, we discuss methodology of constructing of physical theories. Within this methodology, one accentuates the problem how to estimate whether mathematics applied to description of physical processes really reflects reality. To this end status of assumptions introduced for theory which is constructed and status of theory are discussed. The term "status of theory" is introduced instead of the term "true theory". This step allows us to estimate to what degree the theory approximates reality. The term "true" is seen as too radical for estimation of degree of this approximation. Discussion is carried out in context provided by evolving dynamical system which can realize cognition process. Within this approach, implication is associated with action of the dynamical system and by this differs from alternative, conjunction, and negation. We consider status of assumptions with respect to terms induced by dynamical systems, status of assumptions with respect to length of infering leading to introduction of these assumptions and status of theory as such having in mind its universality and fundamental properties. One discusses relation of experimental data and theories which interpret corresponding experimental results. Status of various assumptions and theories of contemporary theoretical
\end{abstract}

2010 Mathematics Subject Classification: 03A10.

Keywords and phrases: methodology of construction of physical theories, fitting of theory to reality, status of implication.

Received January 27, 2016

(C) 2016 Scientific Advances Publishers 
physics is estimated as examples. Role of theories which have low status is considered in context of recognition of reality.

\section{Introduction}

It seems that contemporary theoretical physics produces physical theories without appropriate discussion of methodology which should be applied to construction of these theories. In particular, role of mathematics is not entirely elucidated. We have to do with mathematical physics where attention is concentrated on correct application of mathematics. However, fitting of theory to physical reality is in this case of secondary importance.

One suggests that theoretical physics should consider two aspects: The first one is related to correct application of mathematics within physical theory and the second one is related to quality of fitting of the applied mathematical theory to reality. The second aspect is more related to methodology of creation of physical assumptions.

This paper is devoted to larger degree to the second aspect. How we can recognize which theory appropriately approximate reality. This aspect is especially important when we are not able to test new theory by experiments. In other words, we frequently create hypotheses in regions of reality which are not accessible by our experiments. Then, cognition power of our theory is the most interesting problem.

In order to provide context for discussion of quality of description of reality by theory, we discuss first how theory could appear in our world. To this end, we discuss evolving dynamical system within which logic and mathematics could appear as a result of interaction with external world.

We introduce the notion "status" of assumptions applied to construction of theory and "status" of theory as property, which is responsible for quality of approximation of reality by this theory.

Having at our disposal context provided by dynamical systems, we discuss status of assumptions in relation to terms induced by dynamical 
system as well as in relation to length of proof applied in infering on form

of these assumptions. We discuss also factors which decide on status of whole theory.

In particular, one indicates that we have to do in theoretical physics with tendency to constructing of fundamental and universal theory. Methodology which is helpful for attaining such an end is discussed.

We discuss also various examples where we estimate status of assumptions applied in contemporary theoretical physics $[1,2,3,13]$.

\section{Estimation of Status of Fundamental Assumptions of Theory}

\subsection{Estimation of status of fundamental assumptions with respect to set of terms}

In order to estimate which assumptions lead to theory reflecting reality with possibly the best quality, we should have at our disposal context for thinking on this problem. Such a context is related to way how the cognition process is carried out.

We assume that our brain and whole biological organism is a dynamical system which can evolve. Thereby, we should look for corresponding context for quality of cognition by theory within dynamical systems. We discuss here some aspects of such an approach given in [4].

We introduce here dynamical system which can undergo evolution and is able to generate cognition process. Let us assume namely that our dynamical system contains the following subsystems: the neural network dynamical system $(N)$, motor system $(M)$, sensor system $(S)$, environmental dynamical system $(E)$, and the main dynamical system $(\mathcal{M})$.

All introduced subsystems interact by assumption. Source of activity follows from the main dynamical system. It can interact with environmental dynamical system immediately or by means of neural network, motor and sensor systems. 
We distinguish the following groups of variables of our dynamical system: $x_{E}, x_{N}, x_{M}, x_{S}, x_{\mathcal{M}}$. In particular, for description of interactions, we distinguish subgroups of variables which take part in these interactions directly. They are specified within the following dynamical systems:

$$
\begin{gathered}
\dot{x}_{E}=A_{E}\left(\bar{x}_{E}, x_{E M}, x_{E S}, x_{E \mathcal{M}} ; x_{M E}, x_{S E}, x_{\mathcal{M E}}\right), \\
\dot{x}_{M}=A_{M}\left(\bar{x}_{M}, x_{M E}, x_{M N} ; x_{E M}, x_{N M}\right), \\
\dot{x}_{S}=A_{S}\left(\bar{x}_{S}, x_{S E}, x_{S N} ; x_{E S}, x_{N S}\right), \\
\dot{x}_{N}=A_{N}\left(\bar{x}_{N}, x_{N M}, x_{N S}, x_{N \mathcal{M}} ; x_{M N}, x_{S N}, x_{\mathcal{M N}}\right), \\
\dot{x}_{\mathcal{M}}=A_{\mathcal{M}}\left(\bar{x}_{\mathcal{M}}, x_{\mathcal{M E}}, x_{\mathcal{M N}} ; x_{E \mathcal{M}}, x_{N \mathcal{M}}\right) .
\end{gathered}
$$

Particular groups of variables specify parts of subsystems interacting. We discuss the notation for the first subsystem as example, where $x_{E}=\left\{\bar{x}_{E}, x_{E M}, x_{E S}, x_{E \mathcal{M}}\right\}$. Variables $\left\{x_{E M}, x_{E S}, x_{E \mathcal{M}}\right\}$ are designed to describe interaction with other subsystems. On the other hand, $x_{M E}$, $x_{S E}, x_{\mathcal{M E}}$ represent subgroups of variables of remaining subsystems within the first one also in order to describe corresponding interactions.

We do not specify here how such a dynamical system evolves. This is not necessary for our considerations at this moment. However we accentuate fact of this evolution since it is important for future investigations on this line as well as for further discussion. Consequently, we introduce here an abstraction level for our considerations. Particular determination of the dynamical system could be seen as entirely separate problem and perhaps even branch of knowledge. This could be associated with considerations related to biology. However, corresponding determination of the dynamical system can also have entirely abstract form and can be considered within pure mathematics.

Subsystems are designed to describe particular properties of the whole system. Consequently, the external dynamical system describes 
behaviour of an environment in which the subsystem $S_{A}=S_{\mathcal{M}} \cup S_{N} \cup$ $S_{M} \cup S_{S}$ acts. Driven force for such an action is generated by the subsystem $S_{\mathcal{M}}$. It can interact with the environment directly or realize an action by means of neural network system and motor and sensory system.

Concept of subsystems suggest that $S_{\mathcal{M}}$ represents the highest level of complexity of an autonomous system which can be interpreted as a living organism for instance. It has to survive in the environment. Therefore, this system generates an action. In particular, this action is associated with an attractor defined within the main dynamical system.

Whole system $S_{T}=S_{E} \cup S_{\mathcal{M}} \cup S_{N} \cup S_{M} \cup S_{S}$ is called here the total system. Then, $S_{E}$ stand for environment dynamical system and $S_{A}$ is called here the autonomic dynamical system.

Introduced above dynamical system is intended to describe a kind of brain surrounded with some functional subsystems in an abstract form. In particular, having in mind such a concept, the neural network system is introduced.

Let us mention that the brain functions are modelled by means of neural network systems in literature, see for instance [5, 6]. Neural networks are considered in many books and monographs, see for instance [7].

In this paper, we consider neural networks which are dynamical systems. Consequently, time is important in such considerations. There are many approaches in literature where dynamical properties of neural networks are discussed $[8,9,10,11]$. We do not specify at this moment any detailed form of the neural network dynamical system in order to express our concepts in what follows.

Logic appears when we have at our disposal a system of formulas and ability to determination of the true. In order to construct the system of formulas we have first to define terms [12]. 
Terms have to be considered here within the neural network system. We assume that they are associated with action of motor and sensor systems by means of the functions $\pi_{S N}$ and $\pi_{M N}$. They transform the set of variables of the sensory system $V_{S}$ and motor system $V_{M}$ into set of variables of the neural network system $V_{N}$. Thus, we have $\pi_{S N}: V_{S} \rightarrow V_{N}$ and $\pi_{M N}: V_{M} \rightarrow V_{N}$. Both functions are determined by means of previously introduced dynamical systems (1)-(5).

The set of terms induced by dynamical systems $S_{M}, S_{S}$ is defined by

$$
T=\pi_{M N}\left(V_{M}\right) \cup \pi_{S N}\left(V_{S}\right) .
$$

The set $T$ contains variables associated with motor and sensor systems expressed within the neural network system.

Consequently, terms which will be next applied to generation of whole theory are a stage on this way. At this moment, we could characterize status of fundamental notions applied to a theory constructed and describing an external world, having in mind methods of generation of these fundamental assumptions.

In case of theories describing a reality, we believe that corresponding theories describe well this reality. Therefore, the term "status" of the theory would be better for characterizing quality of approximation of reality. In this case, the term "true" appropriate for mathematical theorems is too radical in case of description of reality associated with external world not entirely recognized and approximated by mathematical theory only.

Consequently, high status of theory means that we believe that the description of reality by corresponding theory is good in some sense. Consequently, it is important for us to estimate which theories have a high status and which have the low one.

First, let us notice that set of terms of theory is interpreted as the simplest sensorial information. This provides us premises for 
methodology of construction of set of fundamental notions for theory describing reality. Fundamental notions should be related to the simplest information following from sensors. However, what is it the simplest information.

One suggests to interpret the simplest information as that one which is associated with maximum engagement of the internal neural network system for interpretation of the sensory results and this interpretation is manifested by relatively simple formulas within the neural network.

Remark 1. Fundamental notions of theory describing reality should be related to maximum engagement by action of the dynamical system and its sensory system in order to obtain results which next can be interpreted as the simplest information by neural network system. Then, status of such a fundamental notion is viewed as high.

The action mentioned in this remark is not defined precisely yet. However, we understand it by intuition since terms are obtained by means of activity of motor and sensor system. This problem will be discussed in next subsection in more details.

Let us discuss an example of high status assumption of theory. We discuss here assumption related to multicomponent vacuum medium mechanics [13-21]. We observe creation of electron-positron pair by experiments what suggests existence of at least two components which seem to undergo separation during this process. On the other hand, electron and positron can annihilate which confirms that the two components can be joined after previous separation.

Within our current experimental possibilities, using maximum efforts is associated with using very complex facilities. Then, we are able to state that some components are separated. We call these components by $a$ and $b$ within vacuum medium [13] correspondingly without any understanding of these component. Consequently, the fundamental notions are then expressed as follows: the component $a$ exists and the component $b$ exists without more particular specification of properties of 
these components. Such fundamental assumptions have high status in description of reality. In other words, it is difficult to impair such a direct observation.

Let us discuss now an assumption on existence of quarks as having low status. We observe directly symmetries in heavy particles. Furthermore, these symmetries are not perfect. Let us mention problem of masses of corresponding particles. Thereby, this information appears in neural network system as complex and therefore has low status. Furthermore, we need inferring on existence of quarks by mathematical considerations which additionally decreases status of quarks. The aspect of infering towards determination of fundamental postulate will be discussed in the next subsection.

\subsection{Estimation of status of fundamental assumptions with respect to logic generated by evolving dynamical system}

When we construct theory then the set of terms $T$ is contained in the set of formulas $F$ [12]. Consequently, the question is how we define next elements of the set $F$. In order to do it, we should have at our disposal relations as well as possibility of taking conjunction, alternative, negation, and implication. They should be expressed within the neural network. Therefore, we should discuss a way on which such notions could happen in the neural network.

Let us notice that conjunction, alternative and negation seem to be relatively simple in order to express them in the neural network. The word "and" is easily understand as a primary notion. Negation appears also in a natural way as opposition to "satisfying a relation". Alternative has a similar level of complexity.

Implication seems to be more complicated and also more important for logic. Its role in proving of the true is crucial. Furthermore, it is not evident directly how to introduce implication. In classical logic, the implication is introduced formally by assumed way of valuation. However, intuition associated with implication suggests a causality. This in turn suggests that implication is not entirely internal notion related to 
the neural network system. It should be associated with processes in the environment induced by the dynamical system $S_{A}$ in order to reflect the causality. In this case, environment play role of real world. Thereby, we should postulate a way for introduction of implication in our dynamical system.

We admit in general evolution of introduced subsystems. In particular, this is the case for the neural network dynamical system. At this moment, we do not discuss particular mechanisms of such an evolution. We expect in general that development attained by this evolution leads to possibility of generation of relations, formulas, and methods of valuation of sentences. In particular, relations could appear as a result of action of the main dynamical system which will be discussed below.

We decide which mechanism of evolution is admitted into considerations. Thereby, we discuss then an abstract system which could generate logic and a kind of thinking. Consequently, using such a way of modelling, we should be motivated by concepts of phenomena which we would like to obtain as properties of our dynamical system. Let us discuss some properties related to logic which could appear during modelling of a reality.

Let $\mathcal{A}_{T}$ be an attractor within the total system $S_{T}$ defined predominantly by $S_{\mathcal{M}}$ and let $x_{T}=\left\{x_{E}, x_{N}, x_{M}, x_{S}, x_{\mathcal{M}}\right\}$ belongs to the set of attraction of this attractor. Then evolution of $x_{T}$ represents various processes undergoing self-organization.

Let us define the external process with respect to the main dynamical system as described by mapping $\pi_{\text {ext }}\left(x_{T}(t)\right)=\left\{x_{E}, x_{N}, x_{M}, x_{S}\right\}(t)$ following from (1)-(5).

Definition 1. Induction of an external state process with respect to the main dynamical system by a process of the main dynamical system $x_{\mathcal{M}}(t)$ immersed in the total state process $x_{T}(t)$ is called action of the main dynamical system. 
Action is manifested by direct interaction of the main dynamical system with environment or by induction of processes in the neural network system and by this induction of action of the motor dynamical system in environment. In the second case, we have to do with induction of a process in environment by $S_{M}$ and next detection of changes in this environment by the sensor dynamical system.

We expect that actions of $S_{\mathcal{M}}$ are frequent sufficiently in order to provide considerable amount of data related to properties of environment. Then, results of each action are remembered within the neural network. When some properties of environment would happen repeatedly, then our model having by assumption property of optimization of energy consuming should manifest more strong remembering of effects corresponding to these properties. By this, we expect generation of relations between state of variables which are present on the input of the motor system and output of the sensor system. Consequently, we suggest generation of relations reflecting properties of the environment, within neural network system. Such relations can be applied for extension of the set of formulas $F$.

Above comments on generation of relations indicate connection with structuralism considered within philosophy of sciences [27]. We see that particular objects in environment are not interpreted. They are reflected within neural network dynamical system where they can be considered having some detected by the system properties only.

Let us introduce here implication by means of the action of the main dynamical system defined above. Thus, we obtain

$$
\left(x_{N M}\right)_{A} \Rightarrow\left(x_{N S}\right)
$$

where $x_{N M}, x_{N S} \in T$ and the symbol ${ }_{A} \Rightarrow$ stands for operation of induction of action $A$ of the system $S_{M}$ by means of states $\pi_{M N}^{-1}\left(x_{N M}\right)$ and appearing of the response $\pi_{S N}\left(x_{S N}\right)=x_{N S}$ from the system $S_{S}$. Let us note that action $A$ has to be considered beyond the neural network dynamical system. 
Let us notice that our implication is indexed by kind of action $A$. Therefore, we have to do here with a family of implications in fact. We can introduce more universal implication as

$$
\left(x_{N M} \wedge A\right) \Rightarrow\left(x_{N S}\right) .
$$

In this case, we have to do with two sentences on the left side of implication. The first one states that $x_{N M}$ takes a value and the second one states that action have a determined kind. Then, implication follows the sentence that $x_{N S}$ takes a determined value which can be written more formally using sentences

$$
\left(\left(x_{N M}=a\right) \wedge\left(A=A_{G}\right)\right) \Rightarrow\left(x_{N S}=b\right),
$$

which is shortly written by (8).

Let us notice that we can also consider action of $S_{\mathcal{M}}$ which happen in neural network only. To this end, we can distinguish subsystems within $S_{N}$. Then, let

$$
S_{N}=\bigcup_{i}\left(S_{N M i} \cup S_{N i} \cup S_{N S i}\right) \cup S_{H},
$$

where $S_{N M i}$ is $i$-th motor subsystem within the neural network, $S_{N i}$ is $i$-th part of the neural network system, $S_{N S i}$ is $i$-th sensory subsystem within the neural network. The $S_{H}$ stands for a part of the neural network able to analyse information obtained by means of $S_{N S i}$ following from action within the system $S_{N i}$. The system $S_{H}$ also generates action in $S_{N i}$. Distinguishing of the subsystems enables us to discuss realization of action of the main dynamical system which is closed within part of the neural network.

Discussed above division of neural network into subsystems enables us to discuss implication similar to that one given by (7) which happens on the interior of the neural network only. We can consider the set of terms in relation to $i$-th subsystem by $T_{i}$. We assume as previously that 
they are associated with action of motor and sensor systems $S_{N M i}, S_{N S i}$ by means of the functions $\pi_{S N i}$ and $\pi_{M N i}$. They transform the set of variables of the sensory system $V_{S i}$ and motor system $V_{M i}$ into set of variables $V_{H}$ of the part $S_{H}$ of the neural network system. Thus, we have $\pi_{S N i}: V_{S i} \rightarrow V_{H}$ and $\pi_{M N i}: V_{M i} \rightarrow V_{H}$.

The set of terms induced by dynamical systems $S_{N M i}, S_{N S i}$ is defined by

$$
T_{i}=\pi_{M N i}\left(V_{M i}\right) \cup \pi_{S N i}\left(V_{S i}\right) .
$$

The set $T_{i}$ contains variables associated with motor and sensor systems $S_{N M i}, S_{N S i}$ expressed within the subsystem $S_{H}$.

Consequently, the implication followed by action within the neural network system can be expressed as

$$
\left(x_{N M i} \wedge A_{N N}\right) \Rightarrow\left(x_{N S i}\right),
$$

where $A_{N N}$ represents a kind of action of $S_{H}$ in the $S_{N i}$.

Let us note that $S_{H}$ represents a system which is able to analyse results of action and transfer of input into $S_{N M i}$. However, the action is induced in fact by the main dynamical system governing the $S_{H}$.

Having at our disposal action in neural network system, we have also an opportunity to comparison of the action $A$ from (9) with an action of type $A_{N N}$ created within the neural network system. Then, a model of reality represented by $A_{N N}$ is produced in case when effects of both actions are the same or similar within the neural network.

We have determined how implication appears within evolving dynamical system. By this we can imagine how proof of a theorem could happen. However, we see also that each step of the proof is associated with an action of dynamical system. In case when this action happens in neural network system, we have the problem to what degree each step of 
the proof fits to reality. Each step is associated with an approximation of reality. Thereby increasing of length of the proof should lower status of formula obtained by this proof.

Frequently, we introduce assumptions for physical theory basing on some proofs following from observed properties. Consequently, we come to the conclusion:

Remark 2. Status of assumptions introduced into theory by means of proofs based on observed properties depends on length of the proof. The longer proof the lower status of assumption. This status could be increased when several independent proofs starting from independent facts would lead to the same conclusion inducing just this assumption.

Let us discuss quarks again. We see that quarks are postulated as consequence of rather long infering leading from symmetry group and potentials towards finding generators of corresponding symmetry group. This rather long way of infering additionally decreases status of quark in comparison with estimation of their status in previous subsection.

Let us discuss assumption based on direct consequence. We have obtained assumption on existence of components $a$ and $b$ within vacuum medium mechanics as having high status. We can infer that separation of $a$ and $b$ needs a critical tension within vacuum medium represented by $\varrho$. Neglecting at this moment what the term "tension" means we find that on the surface of particle which is border of $a$ and $\varrho$ the critical condition for separation of components is satisfied. This direct consequence considered as obtained by short proof makes status of this assumption as high. This assumption is important for description of breaking of charge conservation law within vacuum medium mechanics $[14,15,18,20]$. Therefore, we can expect that breaking of charge conservation law can indeed happen in reality during disintegration of proton into three positrons [18]. 


\section{Estimation of Fitting of Theory to Reality}

\subsection{Relations between internal world of the dynamical system and its external world}

We can discuss some aspects of modelling of reality within the neural network system. To this end, let us consider an action of the main dynamical system in the environment mediated by the neural network. Then, the motor system generates the state of environment by its state determined by

$$
x_{M N}=\pi_{M N}^{-1}\left(x_{N M}\right) .
$$

State of environment and value of the variable $x_{M N}$ generate processes in environment described by dynamical systems:

$$
\begin{gathered}
\dot{x}_{E}=A_{E}\left(\bar{x}_{E}, x_{E M}, x_{E S}, x_{E \mathcal{M}} ; x_{M E}, x_{S E}, x_{\mathcal{M} E}\right), \\
\dot{x}_{M}=A_{M}\left(\bar{x}_{M}, x_{M E}, x_{M N} ; x_{E M}, x_{N M}\right), \\
\dot{x}_{S}=A_{S}\left(\bar{x}_{S}, x_{S E}, x_{S N} ; x_{E S}, x_{N S}\right),
\end{gathered}
$$

with initial conditions for $x_{N M}$ given by (13). The remaining part of initial conditions for $x_{S}$ and $x_{E}$ follow from current state of these dynamical systems during initiation of the motor action.

Consequently, response of the dynamical systems (14), (15), and (16) mediated by sensory system provides the result $\pi_{S N}\left(\left\{x_{S N}\right\}\right)=\left\{x_{N S}\right\}$ in the neural network. We consider set of states $\left\{x_{S N}\right\}$ in this place since response of environment on action discussed above does not need to be unique. Let us denote whole action concisely by

$$
\left\{x_{N S}\right\}=\mathcal{T}_{E}\left(x_{N M}\right) .
$$

Thereby, Equations (14), (15), (16) and functions $\pi_{M N}$ and $\pi_{S N}$ are replaced now by the operator $\mathcal{T}_{E}$. 
Let us assume at this moment that $\mathcal{T}_{E}$ reflects reality which is not known. We can express this reality and thereby the operator $\mathcal{T}_{E}$ by set of relations $\mathcal{R}_{E}=\left\{R_{E k}\right\}$ which are not known but represent formally this reality.

On the other hand, having a set of relations $\mathcal{R}_{I}=\left\{R_{i j}\right\}$ considered within $i$-th neural network subsystem, we could approximate the operator $\mathcal{T}_{E}$ defined by means of external processes with the aid of these relation as $\mathcal{T}_{E N}\left(\left\{R_{i j}\right\}\right)$. Then, we can consider transformation of initial values in the motor system independently also by means of $\mathcal{T}_{E N}$. Then we have

$$
x_{N S i}=\mathcal{T}_{E N}\left(\left\{R_{i j}\right\}\right)\left(x_{N M}\right) .
$$

In this place, we have possibility of comparison of (17) with (18). Let us note that the first transformation is carried out in external world having initial state and final state within neural network system. The second transformation is carried out entirely within neural network system.

Let us also note that the set of relations $\mathcal{R}_{I}=\left\{R_{i j}\right\}$ has been created within neural network system by means of various actions of whole dynamical system. Consequently, we can consider a mapping $\pi_{R}: \mathcal{R}_{E} \rightarrow \mathcal{R}_{I}$, which joins formally external and internal, neural network world.

We can compare results of both transformations (17) and (18) with the aid of a function $\delta\left(x_{N S i},\left\{x_{N S}\right\}\right)$ representing a distance in an abstract form. When this function takes an admissible value than we can assume that external world is appropriately approximated by theory expressed by relations $\mathcal{R}_{I}$. However, we do not know in fact content of the set $\pi_{R}^{-1}\left(\mathcal{R}_{I}\right)$. 
Let us consider the set

$$
\pi_{R}^{-1}\left(\mathcal{R}_{I}\right)=\bigcup_{R_{i j}} \pi_{R}^{-1}\left(R_{i j}\right)
$$

Then we see that content of this set is composed of inverse images of particular relations $R_{i j}$. Let us note that perhaps some relations $R_{E k} \in \pi_{R}^{-1}\left(R_{i j}\right)$ can be removed and $\pi_{R} \circ \pi_{R}^{-1}\left(R_{i j}\right)$ could be unchanged. It means that reality is partly described by $R_{i j}$ only.

We would say that the better approximation of reality, the mapping $\pi_{R}$ is more similar to one-to-one mapping.

This discussion on quality of approximation of reality could be perhaps more precise taking into account whole possible structures of theories. However, in this place we would like to have at our disposal a simplified criterion of such an approximation for further considerations.

Let us assume that some relations of the set $\mathcal{R}_{I}$ are dependent. We express this by relation

$$
\Phi\left(R_{i j}, R_{i k}\right)=0
$$

This dependence means in fact that we have at our disposal reduced number of relations in the set $\mathcal{R}_{I}$. It means also that we consider now one relation $R_{\Phi i p}$ instead of both relations $R_{i j}$ and $R_{i k}$. In particular, $R_{\Phi i p}$ can be identified with $R_{i j}$ or $R_{i k}$. We denote this new set of relations by $\mathcal{R}_{\Phi I}$. Then the mapping $\pi_{R}$ has to be replaced by new one $\pi_{\Phi R}$ with smaller set of values. We assume also that $\pi_{\Phi R}^{-1}\left(R_{\Phi i p}\right)=\pi_{R}^{-1}$ $\left(R_{i j}\right) \cup \pi_{R}^{-1}\left(R_{i k}\right)$. As a result of this, $\pi_{\Phi R}$ is less similar to one-to-one mapping than $\pi_{R}$ just since $\pi_{\Phi R}$ has smaller set of values. Then, we come to the conclusion that description of reality by the new theory associated with $\Phi$ is worse. 
We obtain an example of the dependence of type $\Phi$ when we apply equivalence rules. Then, we identify various type of physical objects as equivalent. Let us mention equivalence of mass and energy or wave particle duality in elementary particle physics. Consequently, we obtain the following remark:

Remark 3. Equivalence laws applied in physics reduce number of degree of freedom in theoretical description of reality. This follows from the fact that corresponding to this situation mapping $\pi_{R}$ becomes then less similar to one-to-one mapping. It means that approximation of reality by theory is worse in case of application of equivalence laws.

We can state that application of equivalence laws decreases resolution of seeing of reality by theory. In other words, we distinguish smaller number of details of reality in this case.

Let us mention some examples. We apply wave particle duality in elementary particle physics. This allows us to avoid definition of elementary particle and treat it as waving phenomenon. This step induces seeing of less number of details in this reality by theory. We can expect that we see less number of details directly near elementary particle since wave has a length. This follows also that quantum field theory has low resolution of seeing of reality in regions placed directly near the particle. Let us note that Higgs particle is not observed directly. This particle is observed by means of observations of other particles, which are considered as products of decay of the Higgs particle. Such an interpretation is related to processes which happen just directly near colliding protons. Above discussion suggests that interpretation that we have to do there with the Higgs particle defined by the Standard Model has low status.

Let us mention concept of virtual particles which follows from equivalence of mass and energy and also form the uncertainty principle. Both premises for the concept of virtual particles are not appropriate methodologically. The first one as equivalence law reduces number of 
degree of freedom in theoretical description and by this makes approximation of reality less precise. The second one, the uncertainty principle, has connections with wave particle duality what increases effect of lowering of resolution of seeing of reality by theory. All this is related to region placed directly near elementary particle where we expect rather increasing of resolution of theory for description of processes placed there. Virtual particles are considered just is such regions. Thereby, we should reject concept of virtual particles due to methodological reasons.

Let us comment some aspects related to status of quantum mechanics. Quantum mechanics is successful theory taking into account its historical step which allow us to consider particles in non-classical way. However, contemporary requirements suggest more important role of dynamics on the most fundamental level. Instead of this quantum mechanics becomes too geometrical and quantization procedure connects the wave function too strongly with classical motion. This is in part just owing to wave particle duality which admits to relinquish defining of elementary particle. Consequently, we come to the following conclusion:

Conclusion. We would say that the most incorrect methodological step in development of quantum mechanics is related to neglecting of description of waving associated with elementary particle as separate phenomenon which is discovered. Instead of this the wave particle duality is applied. The phenomenon of waving should be considered as independent even on elementary particle.

This situation is taken into account in vacuum medium mechanics. Waving is considered as independent phenomenon on elementary particle $[14,15]$. No wave particle duality is considered. Instead of this, we define elementary particle within vacuum medium mechanics as follows: object within vacuum medium which contains at last one rotating part composed of component separated from resting vacuum medium by discontinuity surface is interpreted as elementary particle. 
Thereby elementary particle is not defined by means of wave. In particular, elementary particle can be associated with waving of components in neighbourhood of the particle when it moves. However, in general this is not the case. Neutrino for instance has no wave function [17]. Photon is not elementary particle since it is associated with waving of well localized components only which are not entirely separated from resting vacuum medium.

All this provides context for better description of dynamics on the most fundamental level. This has important consequences for theoretical biology for instance [15, 23]. Owing to better description of dynamics, we are able to describe attractor governing biological evolution and, for the first time, this mechanism of self-organisation is considered on the most fundamental, elemenatary particle level.

Let us note that simplified picture of dynamics of elementary particles represented within quantum mechanics by wave function and associated with it point-like particle follows too easy generalization of physical theories towards larger dimensions. This generalalization is easy since the elementary particle considered as point-like is almost nothing in physical reality. However, when elementary particle is extended and associated with mechanisms of motion as in vacuum medium mechanics for instance then natural generalization to larger dimensions is not so easy. This follows from fact that we have to generalize to larger dimensions also all aspects of dynamics including mechanisms of motion. Summarizing, extension of system of fundamental notions and by this resolution of theory for seeing of reality makes more difficult inductive thinking leading to various forms of generalizations.

\subsection{Properties and status of theory}

Important aspect of constructed theory is related to its range of validity. Usually physical theories have a determined range of validity which is not too large. Let us mention models of particular elementary particle interactions. Range of validity is associated with system of fundamental assumptions applied. The larger system of assumptions the larger range of validity of theory. Fitting of theory to reality in this case depends on status of assumptions applied. 
Another important aspect of constructed theory is associated with fundamentality of theory. We say that theory $T h_{1}$ is more fundamental than the theory $T h_{2}$ when assumptions of the theory $T h_{2}$ can be interpreted or proved within theory $T h_{1}$. We denote this by $T h_{2} \subset_{f} T h_{1}$. This means that number of assumptions of the theory $T h_{1}$ is larger than that one for theory $T h_{2}$. This means also that resolution of seeing of reality by theory $T h_{1}$ is larger than by theory $T h_{2}$.

In general, we would like to have at our disposal fundamental and universal theory with large resolution of seeing of reality by theory. The term "universal" means large range of validity of the theory with respect to description of various aspects of reality. Concisely, we would like to have at our disposal within theoretical physics one theory. Sometimes such a theory is called "theory of everything". The last term is perhaps not appropriate since we do not understand the term "everything". We would like to have at our disposal rather theory with large range of validity as far as it is possible.

We can summarize our discussion in order to estimate which factors are responsible for status of theory with respect to quality of approximation of reality.

Remark 4. We would say that status of theory depends on number of assumptions applied to construction of this theory, status of each assumption with respect to criteria discussed previously, range of validity associated with universality of theory and with status of logic.

The last aspect of the remark is associated with generation of logic within dynamical systems. This aspect is not discussed here in more detail. However, in cognition process we should be aware where from assumed logic appears.

Mentioned above universality is important aspect of theory within theoretical physics. We try to have universal theory as far as possible therefore important question is how to construct universal theories. 


\subsection{Remarks on methodology of constructing of universal theories in physics}

Let us note that it is impossible to construct theory with large range of validity in one step. This is so since corresponding theory should describe large variety of experimental results. Therefore, universal theory should be constructed step by step. The question is how to characterize corresponding steps.

In contemporary physics, we encounter tendency to direct verification of new constructed theory with any accessible experimental results which is interpreted then as success. As a result of this, we obtain large number of theories for various phenomena which have rather small range of validity and are also characterized by different methodologies applied. Let us mention division of elementary particle physics into four fundamental interactions. As a result of this, it is difficult to obtain unified theory since methodologies applied to description of particular interactions are different.

In order to obtain universal theory, one suggests to construct larger number of qualitative models associated with particular phenomena observed and next join them into one theory. Whole process should be characterized by similar methodology for all constructed models. It means that we should have at our disposal a plane for unification still before the models are constructed. Such a plane can be associated with concept of vacuum medium for instance [13].

Let us consider a set of phenomena observed in reality and expressed as properties $\phi_{k}, k \in I_{N}=\{1,2, \ldots, N\}$. Qualitative modelling means that we do not determine all constants within the model but we are concentrated on fact that the model is able to describe qualitatively the chosen property $\phi_{k}$.

Let us mention for instance waving of components within vacuum medium [14] or precession of electron [19]. We could propose perhaps various models of this waving or various models of precession. However, 
at this moment, it is important to obtain models which are able to describe chosen properties without direct scalling of constants which appear within models.

Let us consider the phenomenon within reality expressed by $\phi_{k}$. Then models which describe this phenomenon we denote by $m_{\phi_{k}}$. Consequently, they are equivalent with respect to property $\phi_{k}$. This can be expressed by the formula

$$
m_{\phi_{k} i} \equiv_{\phi_{k}} m_{\phi_{k} j}
$$

The models $m_{\phi_{k} i}, m_{\phi_{k} j}$ describe the same property. However, they can do it on entirely different ways. Then whole equivalence class of corresponding models associated with the property $\phi_{k}$ is denoted by $\mathcal{M}_{\phi_{k}}$. We can introduce also the set

$$
\mathcal{M}_{\phi}=\bigcup_{k} \mathcal{M}_{\phi_{k}} .
$$

The set $\mathcal{M}_{\phi}$ represents all accessible models for various phenomena observed. The question is how to indicate universal theory with the aid of models contained in the set $\mathcal{M}_{\phi}$.

We should do cross-section within the set $\mathcal{M}_{\phi}$. We can define this operation by

$$
\mathcal{C}\left(\mathcal{M}_{\phi}\right)=\left\{m_{\phi_{k} i}: m_{\phi_{k} i} \in \mathcal{M}_{\phi_{k}}, m_{\phi_{k} j}, m_{\phi_{k^{\prime}}} \in \mathcal{C}\left(\mathcal{M}_{\phi}\right) \Rightarrow k \neq k^{\prime}\right\} .
$$

By the formula (23), we have defined the mapping

$$
\mathcal{C}:\left\{\phi_{k}\right\} \rightarrow \mathcal{M}_{\phi}
$$

The mapping $\mathcal{C}$ selects set of models which together describe all chosen properties of reality. However, in order to construct model of all these phenomena we should join selected models. Consequently, by theory of reality composed of all models of $\mathcal{C}\left(\mathcal{M}_{\phi}\right)$ we understand 


$$
\mathcal{C}_{J}=\left\{\mathcal{C}, \mathcal{J}_{\text {PROC }}\right\}
$$

where $\mathcal{J}_{\text {PROC }}$ means procedure of joining of models from $\mathcal{C}$. This procedure can be associated with division of three-dimensional space represented by vacuum medium for instance into parts $V=\bigcup_{m} V_{m}$. Then joining of models can be realized by means of boundary conditions determined on $\partial V_{m}$. The procedure can be also associated with introduction of additional postulates for variables of joined models related to energy for instance.

At the stage of joining of submodels, we should obtain additional premises for determination of constants of models considered.

Above discussion characterizes steps in constructing of universal theories. We see that we are interested in construction of various models belonging to the same equivalence class $\mathcal{M}_{\phi}$. Then corresponding crosssection $\mathcal{C}$ can be carried out within larger context related to modelling of reality. Thereby, going step by step towards universal theory, we determine as an intermediate scientific aim construction of elements of a given equivalence class $\mathcal{M}_{\phi_{k}}$ which next are components of $\mathcal{M}_{\phi}$. In other words, we are interested in speculative modelling of particular properties along a chosen methodology.

Models belonging to $\mathcal{M}_{\phi}$ can be complicated as such. Thereby, we are interested also in constructing simpler models which gradually lead to a given $m_{\phi_{k} i}$. Let us mention some one-dimensional models which describe waving of components for instance. They do not describe reality since the reality is three-dimensional. However, one-dimensional models can be helpful as intermediate step for elaboration of three-dimensional models. Consequently, artificial submodels which do not belong to $\mathcal{M}_{\phi}$, discussed as steps towards obtaining of a given $m_{\phi_{k} i}$ can also be considered as scientific aim. 
Above methodology should lead to classification of various properties $\phi_{k}$ which should be modelled and also to classification of elements of $\mathcal{M}_{\phi}$.

\subsection{Role of experimental data in recognition of reality}

Frequently, we have to do with comments that physics is an experimental science. To some degree this has been the case when physics was in less advanced stage of development. Then interpretation of experimental results was simpleer. However with transition of physics to processes related to smaller scales interpretation of experimental results becomes less clear.

Frequently, we have to do with situation that a theory suggests a particular experiment. Then, obtained within this experiment results are interpreted just by this theory. However, interpretation of experimental results depends on theory applied to this interpretation. Consequently, we can obtain various interpretations of experimental results when we apply various theories.

Let us mention in this place an example. We have observed that all galaxies move away in space. Then, we come to the conclusion that all they was close in the past. This concept leads to Big Bang theory. Contemporary theory suggests that Big Bang is associated with explosion of something very small and gradual creating of matter by creation of pairs process in expanding space. However, another interpretation suggests explosion of space within extermely dense matter [16] and creating space by annihilation of electrons on protons. Then, galaxies are remnants of this dense matter instead to be composed of created matter. Both approaches take into account the same experimental result. However, interpretation is extremely different.

We would say that such a situation is associated with fact that we do

not know content of the set $\pi_{R}^{-1}\left(\mathcal{R}_{I}\right)$, where $\pi_{R}$ is mapping discussed in connection with formulas (17) and (18). 
Above discussion suggests avoiding comments of experimental results without indication of theory by which the interpretation is carried out. Let us mention as example the situation when we obtain information that scientists have discovered that neutrina have mass. This statement is not appropriate. The discovery is related to change of type of neutrina. However, mass is related to interpretation by the Standard Model [1]. Other interpretations [17, 21] suggest that neutrino can change their type but have no mass.

Similar situation is related to quarks. They are not discovered. However, we obtain information that bottom or top quark is discovered. In fact, an elementary particle which is interpreted by the Standard Model as containing corresponding quark is discovered. Appropriate comments frequently are not applied in order to explain this situation.

Summarizing, it is not assumed as custom to accentuate which theory is applied to interpretation of experimental results against fact that corresponding interpretations can differ considerably.

The question is what for experimental results are. First, experimental results can provide unexpected new properties of processes which induces theoretical considerations. Then, experiment is a way of recognition of reality in region where we were not able to carry out experimental investigations previously.

Another task for experimental investigations is related to determination of various constants of qualitative models of physical processes and transition by this to quantitative models.

We can apply experiments also to falsification of theory. When we find results which contradict considered theory then we can reject corresponding theory in accordance with considerations carried out by [22]. However, in the framework of previous discussion on physical theories we can weaken this point of view. Within our discussion, it is accentuated the fact that physical theory approximates reality only and is not identified with entirely precise description of reality. This is 
expressed by status of theory instead of statement that the theory is true. Then, falsification states that theory is not true but it does not indicate that the theory does not approximate of reality. Falsification decreases status of theory. It states in fact that in some region approximation of reality is not appropriate. This statement decreases status of theory. However, the theory could approximate reality correctly in some smaller regions.

Such a situation is related to the Standard Model. Within vacuum medium mechanics quarks do not exist what has considerable support by interpretation of various experiments and cosmological observations $[18,21]$. Such an interpretation of experimental data is possible owing to sufficiently large system of fundamental notions of vacuum medium mechanics and this is not possible within the Standard Model.

Falsification suggests direct rejection of the Standard Model in considered case. However, quarks are introduced by means of really observed symmetries within heavier particles. Consequently, quarks as generators of corresponding symmetry group makes the Standard Model as fitting to reality better than in the case when the concept of quarks would be neglected. However, non-existence of quarks decreases status of the Standard Model as universal theory. We come to the conclusion that falsification of theory should not lead to rejection of theory but to diminishing its range of validity.

The Standard Model is seen today as the most fundamental and universal theory well fitting quantitatively to experimental data. However, within this model qualitative accordance with reality is not maintained. Let us mention fact that quarks do not exist [18] or status of boson $Z$, which is really discovered. The boson $Z$ is not carrier of electroweak interactions [17, 4] against interpretation within the Standard Model. The last property follows from fact that the boson $Z$ is not present in direct interactions of electron and neutrino and concept of virtual particles is not appropriate. This suggests that methodology applied to construction of the Standard Model is not appropriate. We summarize above discussion by the following remark: 
Remark 5. During construction of fundamental and universal theory, we try to ensure first qualitative accordance with experimental data. This means that the most important is existence of a given phenomenon within theory. In the second stage, when existence of all observed phenomena is ensured, we carry out identification of all constants in order to obtain quantitative theory.

This remark shows that we do not tend towards quick fitting of elaborated fundamental and universal theory to quantitative experimental data. This is rather long range aim.

Let us also note that traditionally high precission accordance of theory with experimental data is seen as appropriate. However, let us note that we do experiments in relation to unknown reality. Furthermore, theory cannot be considered as full description of reality. It means that perhaps high precission accordance with experimental results should not be the case or should not be considered as advantage of primary importance. Then qualitative accordance of theory constructed with experimental results is perhaps much more important than today is considered.

\subsection{Role of theories with low status}

Previous discussion on role of experimental data in recognition of reality suggests that physics is not experimental field only. Within transition to smaller scale interpretation of experimental data depends to larger and larger degree on kind of theory applied to this end. Furthermore, it becomes more and more difficult to propose new kinds of experiments. These new experiments have to be suggested by means of a theory perhaps entirely new one. In other words, conjugation of experiment and theory increases with transition to border of cognition.

Such a conclusion suggests directly that theories strongly connected with hypotheses should be more and more important as method of recognition of reality. It seems that construction of more appropriate theory, having higher status, would be preluded by various theories of 
low status. Theories of low status are then associated with hypotheses and their verification provides extension of context for determination of better theory.

We would say that evolution towards theory better fitting to reality $T h_{F}$ is seen as a self organization process

$$
T h_{1} \rightarrow \mathcal{C}_{1} T h_{2} \rightarrow \mathcal{C}_{2} \cdots \rightarrow \mathcal{C}_{F-1} T h_{F}
$$

where $T h_{1}$ is theory considered as starting point for this process.

The question is what circumstances induce transition $T h_{k} \rightarrow_{\mathcal{C}_{k}} T h_{k+1}$. The answer to this question states that each step of transition to better theory is associated with context $\mathcal{C}_{k}$ which provides premises for such a step. Context is associated with comprehensive analysis of theory $T h_{k}$ with respect to its status. We can do it by theoretical considerations only however in relation to interpretation of existing experimental data. The theory can describe processes related to small scale which are not accessible by experimental investigation directly. However, existing experimental data related to larger scale processes for instance can be explained by this theory. Quality of accordance of description of larger scale experiments could lead to increasing of status of this theory or introduction of new postulates. Consequently, with time we can discern deficiencies of theory $T h_{k}$ and produce better additional hypotheses leading to modification of $T h_{k}$ towards $T h_{k+1}$ or a jump to new theory $T h_{k+1}$ by introduction of entirely new system of assumptions.

Let us discuss an example of doing assumptions for theory with low status in region where it is difficult for us to carry out experiments. We consider within vacuum medium mechanics deeper structure of vacuum medium [21] leading to interpretation of previously discussed components $a, b$ and $\varrho$ for larger scale. Let us note that we discuss for this larger scale four components for vacuum medium

$$
\varrho_{v}+\varrho_{\bar{v}}+\varrho_{w}+\varrho_{\bar{w}}=\varrho
$$


The first two are associated with electric field and the next two are associated with magnetic field [13]. Components $a$ and $b$ follow from decomposition of $\varrho=a+b$. In this decomposition, $a$ and $b$ have formal connection with the four components with predominant connection with $\varrho_{v}$ and $\varrho_{\bar{v}}$ correspondingly [13]. In this description, electron is composed of the component $a$ and positron contains the component $b$. All above discussed components are related to scale $S C_{1}$.

We would like to produce theory related to deeper structure of vacuum medium, related to scale $S C_{2}$ smaller than $S C_{1}$ having a hope for better understanding of reality. Consequently, we have to start with assumptions of lower status which are difficult to confirmation by direct experiment. In this case lower status of assumptions is connected with longer infering.

We assume that at the scale $S C_{2}$ the vacuum medium is considered as a stable background for other processes and is composed of three dominant components. The first one is associated with gravitational field h. Next two components are associated with the fields $\mathbf{w}$ and $\overline{\mathbf{w}}$ representing corresponding polarization of components within an unit of vacuum medium and leading to magnetic field $\mathbf{q}=\mathbf{w}-\overline{\mathbf{w}}$. Then, no electric field is considered [21].

We assume furthermore that components $\varrho_{\mathbf{w}}$ and $\varrho_{\overline{\mathbf{w}}}$ related to magnetic field can be separated. These components correspond with $\varrho_{w}$ and $\varrho_{\bar{w}}$ considered for vacuum medium [13] related to scale $S C_{1}$ where they do not undergo any separation. The separation considered here leads to a kind of magnetic monopoles of two kinds. Such objects should be rather of finite size. Therefore we assume, similarly as for electron, that they have spherical form and rotate [19].

Magnetic monopoles introduced above are considered as main components of $a$ and $b$-media as well as $\varrho_{v}$ and $\varrho_{\bar{v}}$ responsible for generation of electric field within $S C_{1}$ scale model. 
Let us note that electron generates electric field since attraction within corresponding components leads to polarization of components $\varrho_{v}$ and $\varrho_{\bar{v}}$ within stable $\varrho$-medium. Discussed above monopoles should not generate of magnetic field at larger distances in perpendicular to surface of monopole direction. Within our concept electron is composed predominantly of magnetic monopoles with component $\varrho_{\mathbf{w}}$ moving with respect to stable background vacuum medium considering scale $S C_{2}$. In case when monopoles would have long range magnetic field in perpendicular direction to their surfaces then the resting electron also should generate static magnetic field in radial direction considered as induced by a magnetic charge. This is not observed. Therefore, within this concept the magnetic field should be suppressed at least at larger distances in perpendicular to the surface of monopole direction.

We assume that the monopole after separation of its component from stable vacuum medium $\varrho_{\mathbf{q}}=\varrho_{\mathbf{w}}+\varrho_{\overline{\mathbf{w}}}$ undergoes rotation and takes shape similar to a sphere. This happens owing to nonlocal attracting forces within each component of the medium and forces which act on the surface of particle. Consequently, we consider monopole particle composed of $\varrho_{\mathbf{w}}$ separated from $\varrho_{\mathbf{q}}$ by a discontinuity surface $\mathcal{S}_{\mathbf{w}}$. Then, motion of this surface corresponds to motion of the monopole.

We have deduced that propagation of magnetic field induced by monopole should be anizotropic. Propagation should have similar properties in various directions placed on tangent to surface of monopole plane and should be reduced in perpendicular to this surface direction. Such a property is introduced by assumption that additional components take part in near-to-surface of monopole processes. They change state of vacuum medium in neighbourhood of monopole which is manifested by new variables.

We introduce the vector $\mathbf{n}_{\eta}$ which can be interpreted as normal to surface of monopole. This vector determines plane tangent to surface of monopole. On this surface the two rings appear as representation of state 
of new assumed components. Furthermore, we consider each ring of the two-ring field as having radius denoted by $\eta_{e}$ and $\eta_{i}$ correspondingly. Consequently, we introduce the variable $\eta$ as follows:

$$
\eta \mathbf{n}_{\eta}=\left(\eta_{e}-\eta_{i}\right) \mathbf{n}_{\eta}
$$

Variables $\eta_{e}$ and $\eta_{i}$ represent radius of a circle associated with new components correspondingly. Circle represents then the same properties on the tangent plane where we do not distinguish any preferred direction. Thereby, both circles are representation of state of new additional components within background vacuum medium in stage close to separation towards magnetic monopole component.

We expect that the same monopoles would attract themselves creating dense fluid composed of monopoles of the same kind what takes place on the inside of electron. On the other hand, opposite monopoles create structure of bounded pairs within background medium with considerably larger distances than monopoles of the same kind within the fluid. This leads to explosion of space after annihilation of electron and positron. As a result of this, we have to organize attraction of monopoles as well as their repulsion in order to ensure relations between mentioned distances [21]. Such a necessity leads just to considering of the two ring field. Then, we obtain possibility that variable $\eta$ can negative and positive values.

The variable $\eta$ has opposite signs on surfaces of opposite monopoles. Consequently, the variable $\eta$ enable us to express anizotropic properties of magnetic field near electron and appropriate interactions of magnetic monopoles [21]. This is done by introduction of appropriate forms of constitutive equations related to the variable $\eta$. This is done also by formulation of critical conditions associated with separation of components which have to be satisfied on surfaces of monopoles and which govern motion of monopoles. 
Above discussed theory is considered in [21] with conclusions in form of the following hypotheses:

Hypothesis 1. Our space is structure composed of pairs of opposite magnetic monopoles bounded on spheres approximately and moving without particular polarization in background vacuum medium. Directional polarization is then associated with electric field.

Hypothesis 2. Magnetic monopoles are the most elementary particles. We consider two kinds of monopoles with opposite sign.

Hypothesis 3. Electron is composed of larger number of magnetic monopoles of the same kind considered as dense fluid which can move within electron during its motion and interactions.

Hypothesis 4. Neutrino is composed of rotating with respect to resting vacuum medium structure of pairs of opposite monopoles.

The theory considered within [21] is not complete yet. The question is how we can increase its status. First of all we should describe observed phenomena in vacuum medium basing on dynamics of monopoles. Let us mention for instance propagation of electromagnetic wave. This phenomenon is seen as quite complicated at this scale and is seen as competition between magnetic field and dynamics of bounded opposite monopoles.

We expect that discussion of various phenomena in above discussed context could provide necessity of introduction of additional assumptions. Other perhaps could be removed. All this should provide extension of context for doing modification of theory to the next one. It is also possible that such an analysis will provide premises for a jump towards entirely new theory neglecting modification of the previous one.

On this level of constructing of theories we have to do with speculative thinking. Therefore, we have to do just with low status theories. The question is whether we are able to maintain discipline during speculative thinking. It seems that introduction of status of 
assumption supports maintaining of such a discipline. Let $\operatorname{St}\left(A_{1}\right)<\operatorname{St}\left(A_{2}\right)$ means that status of assumption $A_{2}$ is higher than status of $A_{1}$. Let us consider the following remark:

Remark 6. Speculative thinking needs changing of assumptions within the same theory. We should introduce hierarchy of assumptions by estimation of their status. Consequently, let $\operatorname{St}\left(A_{1}\right)<\operatorname{St}\left(A_{2}\right)<\ldots<\operatorname{St}\left(A_{N}\right)$ be set of assumptions of a given theory ordered with respect to their status. Then, we should first change assumptions of the lowest status during speculations and gradually do it going towards the largest status assumption $A_{N}$. Consequently, $A_{N}$ should be the most stable during the speculative thinking. It means also that introduction of extra assumptions which makes constructing of theory more simple is interpreted as lack of discipline. We can admit sometimes corresponding extra assumptions. However, we should assign to them sufficiently low status.

Above remark is introduced in order to accentuate fact that even in speculative thinking we are able to maintain a discipline and responsibility.

At this moment, it would be appropriate to discuss concept and role of paradigm [28] in theoretical physics. We would say that a paradigm could be represented by a theory. Then, such a dominant theory could be changed as a result of new established facts what corresponds to a kind of revolution. Let us note that such a step is similar to procedure discussed in (26). We change there theory as a result of investigation of context of this theory. Within this context, we consider new experimental results and their interpretations. However, we consider there also whole system of fundamental notions and hypotheses related to them having in mind estimation of status of corresponding notions. 
Consequently, within this work we come to the following conclusion:

Conclusion. Within this work, one admits and suggests less radical steps than changing of a paradigm. We admit rather evolution of theoretical description towards better one by estimation of its status. Consequently, within this evolution theories which become less appropriate are not removed. Instead of this, they obtain new lower status which characterizes to what degree they approximate a reality taking into account new larger context. This lower status can be expressed frequently by more precise determination of range of validity of corresponding theories.

We see that introduction of status of theory does not leed to direct rejection of theories after falsification. We have tendency to more precise determination of range of validity of corresponding theories. This range of validity is frequently reduced when status of theory becomes lower. However, theories with lower status can be useful. It happens especially in the case when new theories with better system of fundamental notions are not sufficiently developed yet. Then old theories cannot be entirely replaced.

\section{Problems Related to Cognition Process within Dynamical Systems}

We have shown possibility of realization of cognition process within evolving dynamical systems. In particular, theories reflecting reality could be created within such dynamical systems. However, at this stage of considerations the situation is not entirely clear yet. This is so since specification of corresponding dynamical systems is not too simple. This can be done in an entirely abstract form. Then, we could obtain a scenario how cognition process could be realized within pure mathematics.

We can do it also in context of biological organisms. However, biological organisms as dynamical systems which realize cognition are extremely complex. Furthermore, introduction of mechanisms of biological 
evolution is also difficult problem. Then, problem of determination of corresponding dynamical system is associated with development of theoretical biology and corresponding evolution theory for biological organisms. This could be done on basis of vacuum medium mechanics for instance which has been formulated also to this end [15, 23, 24].

Qualitative discussion carried out in this paper can be considered as starting point for more detailed specification of objects considered here and related to form and properties of dynamical systems.

Above discussion provides also context for formulation of new problems. Let us consider evolving dynamical system $D S_{1}$ which recognize external world. As a result of this some laws related to existing reality are established. Corresponding laws create theory $T h_{1}$ which could be next applied to description of this dynamical system again. Let

$$
D S_{2}=\left\{D S_{1}, T h_{1}\right\}
$$

be dynamical system described by action of $D S_{1}$ which rests on application of theory $T h_{1}$ to this end. We could repeat this procedure again and again. As a result of this, we can consider

$$
D S_{n+1}=\left\{D S_{n}, T h_{n}\right\}
$$

An interesting problem is then related to degree of loss of quality of description in each step of this procedure. In other words to what degree organism can describe itself. Can we characterize conditions which would prevent loss of quality of description in particular steps of this procedure.

It seems that methodology of creating of assumptions is important within such a context. Status of logic should also be discussed in more details. It seems that within this context in a future Gödel theorems could be discussed in order to elucidate problem of number of assumptions of theories and complexity in mathematical sense which can be attained. All this should be useful for estimation of cognition power of our brain considered as segment of a dynamical system. 
Let us also notice that presented here approach to generation of logic and theory of a reality is placed within dynamical system especially owing to reinterpretation of status of implication. Consequently, implication considered as associated with action of a dynamical system can lead in a future to creation of physical foundations of mathematics.

\section{Final Remarks}

Methodology discussed in this paper is elaborated during formulation of vacuum medium mechanics. During this process, the fundamental question has been related to better recognition of reality in order to overcome dominant position of quantum mechanics. It is estimated that the quantum mechanics does not describe sufficiently well dynamics on elementary particle level. As a result of this contemporary requirements for fundamental physical theory and related to possibility of description of biological evolution or source of precission for nanotechnological processes cannot be solved.

Speculations leading to formulation of vacuum medium mechanics have been carried out with discipline which is in particular expressed by Remark 6. Furthermore an important aim has been related to construction of fundamental and universal theory. As a result of this, vacuum medium mechanics in present stage of development has

considerable predictive power. It predicts among others existence of bounded state of electron and neutrino as elementary particle with mass placed between mass of electron $0.5 \mathrm{MeV}$ and mass of muon $105 \mathrm{MeV}$ [21].

In last years boson $\mathrm{E}(38)$ has been discovered with mass $38 \mathrm{MeV}$ $[25,26]$. This new particle does entirely not fit to the Standard Model and is not predicted by this theory. Such a fact supports considerably presented in this work methodology of constructing of physical theories as applied in practice. 
Summarizing, in this work, we have discussed methodology of construction of physical theories having in mind especially aspect of fitting of theory to reality. Conclusions are important. It seems that theories within theoretical physics treat problem of status of assumptions superficially. As a result of this considerable freedom in creation of various theories in theoretical physics takes place.

It seems that we need in theoretical physics stronger accentuation of connections of experimental data and status of theories which explain corresponding experimental results. One suggests also that estimation of status of assumptions and corresponding physical theories should become systematic procedure supporting creation of description of physical reality.

\section{References}

[1] C. Itzykson and J.-B. Zuber, Quantum Field Theory, McGraw-Hill Book Co., 1985.

[2] A. S. Dawydow, Quantum Mechanics, PWN Warszawa, 1969.

[3] J. D. Bjorken and S. D. Drell, Relativistic Quantum Fields, McGraw-Hill Inc., 1965.

[4] J. Kaczmarek, On emergence of intelligence in biological systems and methods of modeling of reality necessary for development of theoretical biology, Far East Journal of Applied Mathematics 75(2) (2013), 101-150.

[5] D. J. Amit, Modeling Brain Function: The World of Attractor Neural Networks, Cambridge University Press, 1989.

[6] R. J. MacGregor, Neural and Brain Modeling, Academic Press, Inc., 1987.

[7] S. Haykin, Neural Networks, (1999).

[8] S. Grosberg, Nonlinear difference-differential equations in prediction and learning theory, Proceedings of the National Academy of Sciences USA, 58 (1967), 1329-1334.

[9] M. A. Cohen and S. Grosberg, Absolute stability of the global pattern formation and parallel memory storage by competitive neural networks, IEEE Transactions on Systems, Man, and Cybernetics SMC-13 (1983), 815-826.

[10] I. J. Hopefield, Neurons with graded response have collective computational properties like those of two-state neurons, Proceedings of the National Academy of Sciences USA, 81 (1984), 3088-3092.

[11] W. Maass and C. M. Bishop (eds), Pulsed Neural Networks, The MIT Press, Cambridge, Massachsetts, London, England, 1999.

[12] W. A. Pogorzelski, Classical Functional Calculus, PWN Warszawa, 1981. 
[13] J. Kaczmarek, Speculative mechanics: A concept for modelling the vacuum medium, Physics Essays 12(4) (1999), 709-732.

[14] J. Kaczmarek, Concept of the chain state of particles with the same charge, Advanced Studies in Theoretical Physics 3(1-4) (2009), 13-33.

[15] J. Kaczmarek, On the role of the electronic chain state for evolution of molecular systems leading to a protocell, Advanced Studies in Theoretical Physics 4(9) (2010), 413-435.

[16] J. Kaczmarek, Processes related to black hole state described within four-component vacuum medium model, Advanced Studies in Theoretical Physics 3(1-4) (2009), 35-63.

[17] J. Kaczmarek, A prototype model of the free neutrino, Advanced Studies in Theoretical Physics 2(5-8) (2008), 13-33.

[18] J. Kaczmarek, Arguments supporting model of three-positron structure of the proton, Advanced Studies in Theoretical Physics 5(2) (2011), 63-75.

[19] J. Kaczmarek, Surface superuidity property as a mechanism responsible for rotation of the electron, Advanced Studies in Theoretical Physics 6(28) (2012), 1355-1369.

[20] J. Kaczmarek, A model of electric field in the vicinity of charged particle, Advanced Studies in Theoretical Physics 7(24) (2013), 1165-1187.

[21] J. Kaczmarek, On structural classification of elementary particles within vacuum medium mechanics, Malaysian Journal of Physics 36(1) (2014), 1-19.

[22] K. Popper, The Logic of Scientific Discovery, Taylor and Francis e-Library ed., 2005.

[23] J. Kaczmarek, On a structure of mathematical theory which reflects multiscale aspects and integrity of biological systems I, Far East Journal of Applied Mathematics 76(2) (2013), 79-117.

[24] J. Kaczmarek, On a structure of mathematical theory which reflects multiscale aspects and integrity of biological systems II, Far East Journal of Applied Mathematics 77(2) (2013), 77-111.

[25] E. van Beveren and G. Rupp, Material evidence of a 38MeV boson, arXiv:1202.1739 [hep-ph].

[26] Kh. U. Abraamyan, A. B. Anisimov, M. I. Baznat, K. K. Gudima, M. A. Nazarenko, S. G. Reznikov and A. S. Sorin, Observation of the E(38)-boson, arXiv, (2012).

[27] W. Balzer, C. U. Moulines and J. D. Sneed, An Architectonic for Science, The Structuralist Program, Dordrecht: Reidel, 1987.

[28] T. S. Kuhn, The Structure of Scientific Revolutions, University of Chicago Press, 1962. 